\title{
High-performance Diagnosis of Sleep Disorders: A Novel, Accurate and Fast Machine Learning Approach Using Electroencephalographic Data
}

\author{
Ricardo Buettner \\ Aalen University \\ ricardo.buettner@hs-aalen.de
}

\author{
Annika Grimmeisen \\ Aalen University \\ annika.grimmeisen@studmail. \\ htw-aalen.de
}

\author{
Anne Gotschlich \\ Aalen University \\ anne.gotschlich@studmail. \\ htw-aalen.de
}

\begin{abstract}
While diagnosing sleep disorders by physicians using electroencephalographic data is protracted and inaccurate, we report promising results from a novel, fast and reliable machine learning approach. Our approach only needs an electroencephalographic recording snippet of 10 minutes instead of eight hours to correctly classify the disorder with an accuracy of over 90 percent. The Rapid Eye Movement sleep behavior disorder can lead to secondary diseases like Parkinson or Dementia. Therefore, it is important to classify the disorder fast and with a high level of accuracy - which is now possible with our approach.
\end{abstract}

\section{Keywords:}

Rapid Eye Movement sleep behavior disorder, Random Forest, Spectral analysis, Electroencephalography, Machine Learning

\section{Introduction}

Driven by increases in computational power and the availability of huge new datasets, IT-based healthcare has undergone a dramatic upswing in the past years $[1,2]$. The field has witnessed spectacular advances in the ability of machines to understand data and this can be accompanied by extraordinary successes in medicine, in particular for diagnosing diseases [3, 4] or detecting higher orders of medical substance interactions [5]. The application of most modern machine learning using big data within the healthcare domain fosters this success [1-5].

Despite the progress in this research field, its application to the treatment of sleep disorders, one of the most frequent disorders, is still missing.

The International Classification of Sleep Disorders (ICSD-3) divides sleep disorders into seven classes. In this work we will focus on Rapid Eye Movement sleep behavior disorders (RBD). RBD belongs to parasomnia dysfunctions and can be identified by a loss of muscular atonia during the rapid eye movement (REM) sleep stage [6-8]. Abnormal or disruptive behavior occurs during the REM sleep phase resulting in sleep interruptions and physical injuries like bruises, abrasions, and patients shouting or punching [9]. For the mental and physical health, sleep is an important and critical human process [10]. RBD affects approximately $0.4-0.5 \%$ of the world population, which is about 35 million people, and appears mainly in men over 60 years old [11-12]. To suffer under a secondary disease means a high risk for people with the RBD disorder. About $50 \%$ of all patients will develop another neurodegenerative disease like Dementia or Parkinson within 12 years [13]. To diagnose RBD, polysomnographic recordings (PG) are used. The PGs include, at least, electroencephalography (EEG), electromyography (EMG) and electrooculography (EOG). The EEG is considered relevant for identifying the RBD sleep behavior disorder, requires usually eight hours of recording time which is recorded overnight. Electroencephalography is widely used in medicine and records the neuronal activities of the brain. It is a technique for imaging electrical signals in the brain. The analysis of overnight EEG recordings is complex and time-consuming. In addition, there is the fact that different physicians make different diagnoses using the same EEG recording [13-16].

Spectral frequencies of EEG data can be divided into different frequency bands. These frequency bands were collected with their related frequency bandwidth in table 1.

\begin{tabular}{|c|c|l|}
\hline $\begin{array}{c}\text { Frequency } \\
\text { Band }\end{array}$ & $\begin{array}{c}\text { Frequency } \\
\text { range in } \mathrm{Hz}\end{array}$ & Characterization \\
\hline Delta & $0.5-3.5 \mathrm{~Hz}$ & Deep sleep \\
\hline Theta & $3.5-7.5 \mathrm{~Hz}$ & Sleep and dream \\
\hline Alpha & $7.5-12.5 \mathrm{~Hz}$ & Relaxed awake \\
\hline Beta & $12.5-30 \mathrm{~Hz}$ & Inner restlessness, stress \\
\hline Gamma & $>30 \mathrm{~Hz}$ & Extreme concentration \\
\hline \multicolumn{3}{|c|}{ Table 1: EEG frequency bands }
\end{tabular}


In the literature the exact thresholds of the bandwidths vary. While traditional approaches use roughly aggregated delta, theta, alpha, beta, and gamma spectral powers (see table 1, [17]), we make use of a more sophisticated fine-graded analysis of EEG sub-bands and develop an algorithm correctly classifying RBD sleep behavior disorder with an outstanding accuracy of over 90 percent.

After following the design-science approach [18], we rigorously evaluate our algorithm using unseen data and 10-fold cross-validation on unseen testing data.

Our algorithm outperforms the current benchmark by Islam et al. [15], who achieved a mean accuracy of $70.4 \%$ using support vector machines.

The most important contributions of this paper are:

1. We developed an automatic classification method for an accurate and reliable classification of RBD and non-RBD subjects with an accuracy of over 90 percent.

2. We used 10 minute snippets instead of 8 hour recordings to enable a fast analysis.

3. We present the important frequency bandwidths from $0.5-50 \mathrm{~Hz}$ for classifying the REM sleep behavior disorder. These include the most important frequencies in the subbands of delta (0.5$2.5 \mathrm{~Hz})$, theta $(6.5-8 \mathrm{~Hz})$ and alpha $(10.5-11 \mathrm{~Hz}$, $11-12 \mathrm{~Hz})$.

4. We demonstrate that the spectral power of RBD compared to non-RBD is higher for the first frequencies and lower for the last frequency per subband for all predictive bands.

The paper is organized as follows: Next we describe the data used in our study and the machine learning method. After that, we present the results of our implemented method and discuss it including theoretical and practical implications. Finally, we outline the limitations of our work and make proposals for future work.

\section{Method}

\subsection{Preprocessing}

The dataset used in this paper was collected at the Sleep Disorders Center of the Ospedale Maggiore of Parma, Italy. This paper is focused on polysomnographic recordings from healthy subjects and subjects with the RBD disorder. RBD was professionally diagnosed by doctors according to the standards of the American Academy of Sleep Medicine (International classification of sleep disorders, 3rd edition; equivalent to ICD-10 G47.52). The age of the RBD patients is ranged from 58 to 82 with $86 \%$ male participants and $14 \%$ female participants. The age of the healthy patients ranged between 23 and 42 with $44 \%$ male and $56 \%$ female participants. The data set includes 22 patients with Rapid Eye Movement sleep behavior disorder and 16 healthy subjects. The data is derived from different electrodes scanned at $512 \mathrm{~Hz}$. Each EEG recording lasts about eight hours and was recorded overnight. The full dataset is available from doi:10.13026/C2VC79.

Fig 1 shows the placement of the sensors corresponding to the 10-20 international system.

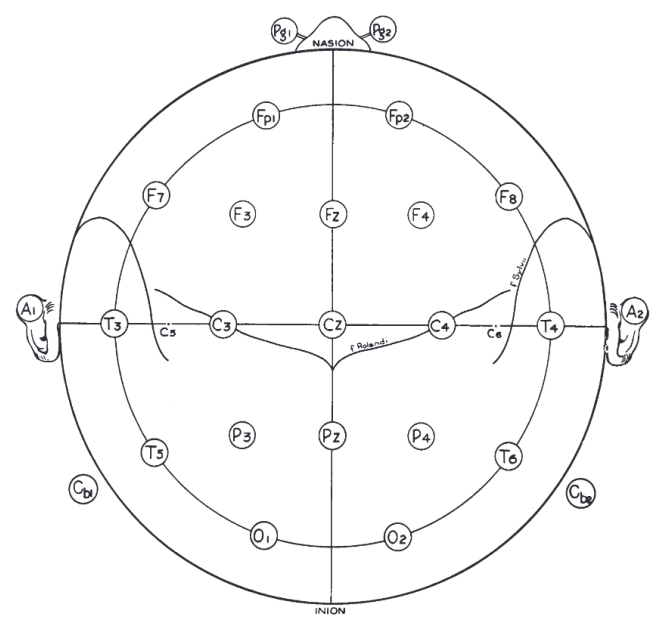

Figure 1: Electrode placement [19]

The extracted signals recorded through various electrodes during an EEG are contaminated by noises which should be removed before further processing. These undesired noises occur during recording and can be divided by noncortical biologic artifacts and environmental noises. Eye blinks or movements and cardiac or muscle activity can be assigned to the group of biological artifacts which are the principal problem.

The appearance of environmental noises can be described with line noises, radio or electrical interference [20]. The blind source separation (BSS) is, next to the Regression, Wavelet transform, empirical mode decomposition and others, one of the most commonly used methods to detect and remove noises. A technique based on BSS is the independent component analysis (ICA). The aim is to extract statistically independent source signals from a linear mixture of observed signals. For application, several premises have to be fulfilled. The principals are that the source signals must be statistically independent and the number of sources has to be equal or lower to the observed signals [21]. Figure 2 illustrates the ICA application on the original EEG (left) and as a result the corrected EEG (right) [22]. 


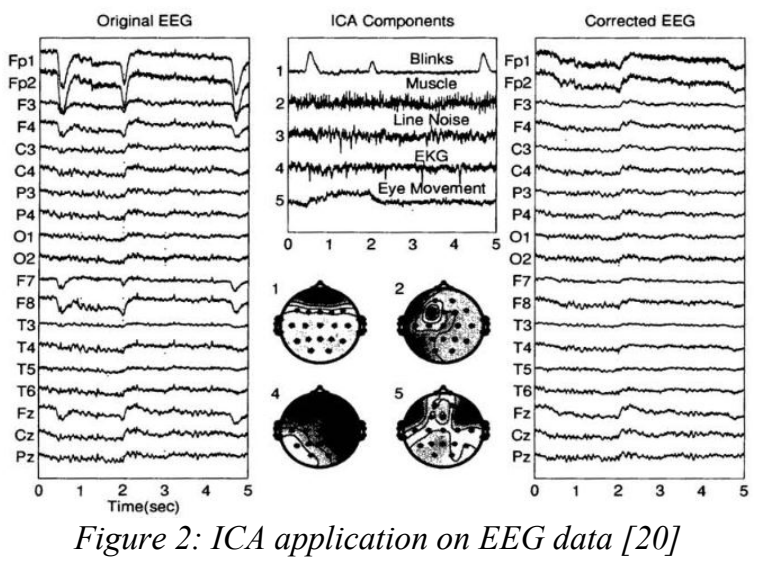

\subsection{Machine Learning Method}

Figure 3 introduces the plan of procedure of this paper. First, the EEG dataset of healthy subjects and subjects with REM sleep behavior disorder is read in the system. Subsequently, the ICA will be performed to clean up the EEG data from environmental noises and noncortical biologic artifacts. The corrected EEG which is now without undesired noises will be transformed by the Fast Fourier Transformation (FFT). Afterwards a classification algorithm is implemented with $75 \%$ training and $25 \%$ testing data. In this paper Random Forest is used to classify RBD from healthy subjects. For validation, the 10 -fold cross-validation is chosen to evaluate the Random Forest classifier to evaluate using unseen testing data.

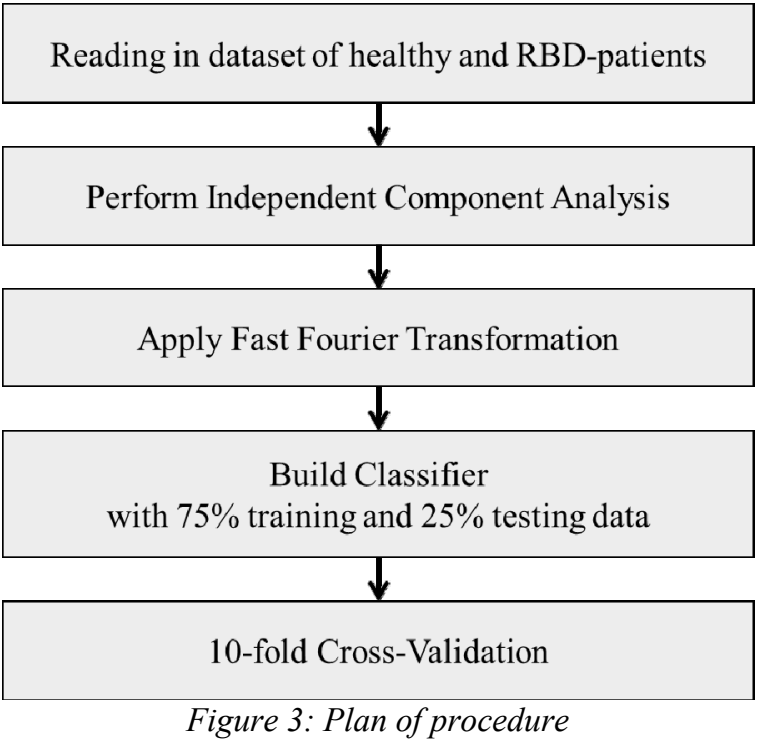

2.2.1. Spectral Analysis. The aim of this work is to determine these frequency bands from the underlying EEG dataset, which are the most important for distinguishing between healthy and RBD-sick patients. For this purpose, this paper refers to the method by [source blinded]. In this method the standard division of the frequency bands in "alpha", "beta", "theta", "delta" and "gamma" was not considered. As feature extraction criterium, the frequency range from 0.5 to $50 \mathrm{~Hz}$ was selected. This range was divided into 99 frequency bands, each of them with a length of $0.5 \mathrm{~Hz}$. With the help of this finer classification, the information content of the individual frequency bands should be increased. Consequently, the level of accuracy for the determination of RBD-sick and healthy patients should be increased too.

After reading and cleaning the EEG data with the ICA, the next step is to transform the data with the help of the spectral analysis. Thereby the signals of the EEG data are converted into sinusoidal frequency components. The method used for the spectral analysis is the Fast Fourier Transformation (FFT). The FFT is a fast, widespread method to determine spectral parameters from neurological signals, which allows an estimate to be made of the frequency components from the signals [23-26]. Figure 4 shows the process of the spectral analysis in simplified form. While the EEG signal is unchanged in the first step, it is converted into sinusoidal signals in the second step. The third and last steps show the individual frequency components from the EEG data.
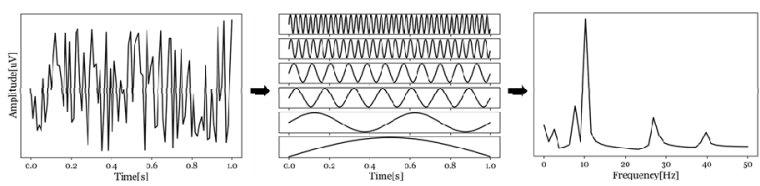

Figure 4: Fourier Transformation EEG signal

2.2.2. Classification by Random Forest. For classification using machine learning, there are a lot of algorithms to use. For instance, support vector machines, neural networks or k-nearest neighbors achieve good results, but they offer no indications about the most important predictors. Due to this fact classification trees (CTs) are the better choice for interpretation. To avoid the sensitivity of small changes regarding the learning sample and so increase stability, an extension of CTs will be used [27]. Ensemble learning leads to a better reliability in an algorithm by using many classifiers. Afterwards the results of these classifiers will be aggregated. Two methods of ensemble learning are prominent: boosting and bagging. By using the bagging method, the algorithm constructs successive independent trees with different separation of data. The result is a ranked list of the most commonly used predictors. For the classification in this paper, the Random Forest approach, which is based on the bagging method, is 
applied. Here, an additional layer of randomness is added to split each node by the best of a randomly chosen subset of predictors instead of all variables [28]. Due to the number of various predictors as a result of the different trees overfitting can be reduced. By this and by handling large amount of data efficiently, Random Forests are very effective at predicting [29]. Furthermore, an advantage of this algorithm is the receiving of information about the importance of the variables. The importance of the variable depends on the rising prediction error when data for that variable is adjusted while others are not [28]. To determine the most important frequencies for the classification of the data used the results regarding the variable importance of the algorithm are needed. Random Forest algorithm works as follows [27, 28]:

1. The original learning sample is divided into $b$ bootstrapped samples $(b=1, \ldots, B)$ while on each bootstrapped sample a tree is grown.

2. For each sample $b$ a random sample $m$ of predictors is taken instead of all variables p. Find the best split among these predictors of the random sample $m$ and repeat this until a maximally sized tree is grown.

3. Aggregate the predictions of all bootstrapped samples $b$ and obtain the majority votes for classification.

The recordings were divided between $75 \%$ training (972) and 25\% testing (324) data, each with 10 repetitions and $n=100$ number of trees. The outcome of the algorithm is the confusion matrix, performance indicators and the calculated variable importance.

2.2.3. Validation. To evaluate the performance of a classifier, the prediction errors of the model need to be estimated. In the machine learning sector, the accuracy is used as a standard measurement to determine the performance of a model. Therefore, there are a lot of methods available like Hold-Out, Bootstrap or crossvalidation $(\mathrm{CV})$. To evaluate the Random Forest classifier used in this paper, the $\mathrm{k}$-fold cross-validation is performed, using 10-folds CV [30-31]. The k-fold cross-validation separates the underlying data set randomly in $\mathrm{k}$ subsets of equal size. Every fold is used in turn for testing the model. The k-1 folds are used as the training data set and the prediction error is estimated with the remaining fold [32].

In order to determine which subjects were classified correctly and which were wrongly classified, the cross-validation Matrix was used. Table 2 shows the matrix, which is differentiated between true positive, false negative, false positive and true negative. If the subject is classified as true positive, it is an RBD patient who has been correctly classified as sick. False negative includes subjects who have RBD but have been incorrectly classified as non-RBD. Under false positive, a healthy patient has been classified incorrectly as an RBD subject. True negative means a healthy patient is correctly classified

\section{Results}

The Random Forest Model was trained with 715 RBD and 257 non-RBD recordings. For testing, 245 $\mathrm{RBD}$ and 79 non-RBD recordings were used. Here, the 10-times cross-validation as well as an electroencephalography frequency bandwidth of 0.5 $\mathrm{Hz}$ were considered. Table 2 contains the results of the model in the form of a confusion matrix. It delivers a total accuracy of 90.12 percent (balanced accuracy $83.13 \%$ ). In addition to the accuracy, table 3 contains other relevant performance indicators. The model was trained with mtry $=100$ and ntry $=50$.

\begin{tabular}{|c|c|c|c|}
\cline { 3 - 4 } \multicolumn{2}{c|}{} & \multicolumn{2}{c|}{ Reference } \\
\cline { 3 - 4 } \multicolumn{2}{c|}{} & RBD & Non-RBD \\
\hline \multirow{2}{*}{ Predicted } & RBD & $\mathbf{2 3 7}$ & 24 \\
\cline { 2 - 4 } & Non-RBD & 8 & $\mathbf{5 5}$ \\
\hline
\end{tabular}

Table 2: Confusion Matrix

\begin{tabular}{lr} 
Performance Indicators & Value \\
\hline Accuracy & $90.12 \%$ \\
True positive rate / Recall & $96.73 \%$ \\
True negative rate & $69.62 \%$ \\
Positive predictive value / Precision & $90.80 \%$ \\
Negative predictive value & $87.30 \%$ \\
Balanced accuracy & $83.18 \%$
\end{tabular}

Table 3: Performance Indicators

\begin{tabular}{l|l|l|l|l}
$\mathbf{H z}$ & RBD & $\begin{array}{l}\text { non- } \\
\text { RBD }\end{array}$ & p-value & $\begin{array}{l}\text { Coh- } \\
\text { ens d }\end{array}$ \\
\hline $0.5-1$ & 0.110523 & 0.042652 & $<0.001$ & 0.267 \\
\hline $1-1.5$ & 0.070853 & 0.039451 & $<0.001$ & 0.392 \\
\hline $1.5-2$ & 0.013933 & 0.007716 & $<0.001$ & 0.298 \\
\hline $2-2.5$ & 0.004044 & 0.007293 & $<0.001$ & 0.209 \\
\hline $6.5-7$ & 0.006464 & 0.001621 & $<0.05$ & 0.950 \\
\hline $7-7.5$ & 0.006158 & 0.003465 & $<0.001$ & 0.997 \\
\hline $7.5-8$ & 0.002781 & 0.005250 & $<0.001$ & 0.766 \\
\hline $10-10.5$ & 0.007014 & 0.005478 & $<0.001$ & 0.824 \\
\hline $11-11.5$ & 0.014493 & 0.010204 & $<0.001$ & 0.378 \\
\hline $11.5-12$ & 0.003423 & 0.012149 & $<0.001$ & 0.516
\end{tabular}

Table 4: Spectral power in predictive subbands 
In table 4 further statistical evaluations of the results are shown. It contains the most important frequencies with their spectral power of RBD and nonRBD. Furthermore, the respective p-value and Cohens $\mathrm{d}$ are calculated. Figure 5 visualizes the spectral power for the most important frequencies per frequency subband. Here, a pattern in each subband can be detected. The spectral power of RBD is always higher for the first variables than the power of non-RBD. In contrast to that, the power of the last variable of RBD is always lower than the spectral power of non-RBD.

\section{Spectral}

Power

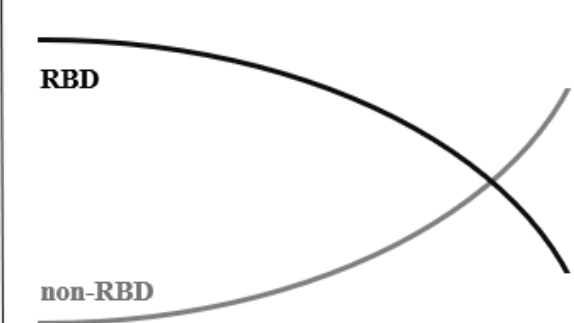

Frequency Subband

Figure 5: Illustration of spectral power per subband

Figure 6 shows the variable importance scaled to 100. The most important frequency band is $1-1.5 \mathrm{~Hz}$ with a relative importance of 100 .

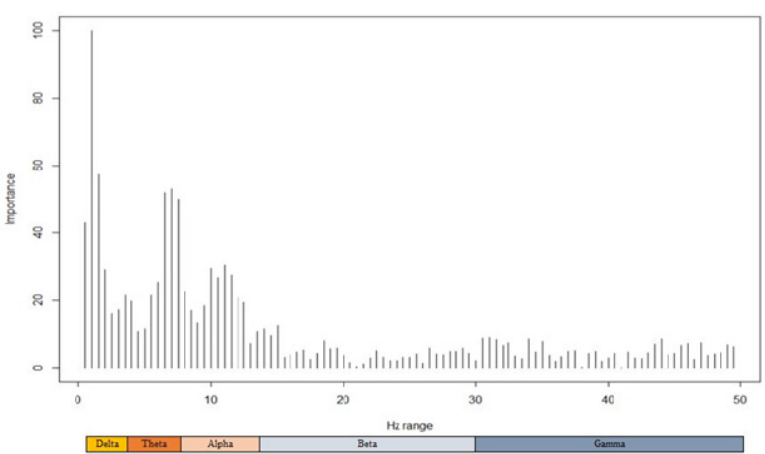

Figure 6: Variable Importance

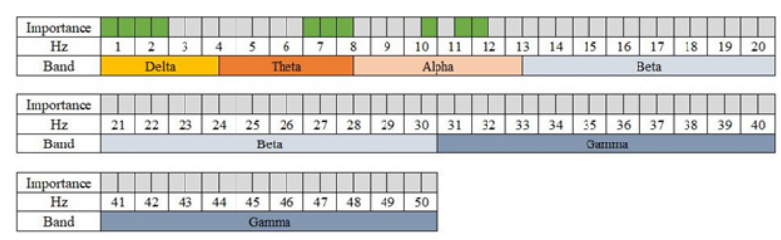

Figure 7: Most important variables

As shown in the figure, the importance of the frequency bands decreases with a higher $\mathrm{Hz}$ range. Figure 7 contains the Top 10 of the variable importance calculated by the algorithm. The following results were achieved: the bandwidth from $1.5-2 \mathrm{~Hz}$ has a value of 57.24 and the frequency band $7-7.5 \mathrm{~Hz}$ a value of 52.99. Further results: $6.5-7 \mathrm{~Hz} 51.89,7.5-8$ $\mathrm{Hz} 49.94,0.5-1 \mathrm{~Hz} 43.22,11-11.5 \mathrm{~Hz}$ 30.43. The bands from 10-10.5 Hz reached a value of 29.61, 2-2.5 $\mathrm{Hz}$ of 29.24 and $11.5-12 \mathrm{~Hz}$ 27.65. When the frequency bands are no longer considered in $0.5 \mathrm{~Hz}$ width but in the standard division delta, theta, alpha, beta and gamma, the ten most important variables are in the frequency bands delta, theta and alpha. The delta band holds four variables, theta and alpha each contain three variables (green-marked in figure 7).

\section{Discussion}

This paper reports on a novel, fast and reliable machine learning approach. Our approach only needs an electroencephalographic recording snippet of 10 minutes instead of eight hours to correctly classify the Rapid Eye Movement sleep behavior disorder with an accuracy of 90.12 percent. The frequency bands of the EEG data are divided into $0.5 \mathrm{~Hz}$ ranges, the standard classification into delta, alpha, theta, and gamma is not considered. Regarding current research, there are various studies that deal with the subject of REM sleep behavior disorder in relation to EEG data. The slowing of waking activity occurs in people who have the REM sleep behavior disorder. In the slowing of waking activity, the theta and delta frequency bands are considered important in RBD-sick patients [33]. Gagnon et al. [34] conducted a study investigating the association of this activity with the risk of RBD patients suffering further cognitive impairments.

Again, a high power of the theta frequency band could be detected. The analysis of EEG data showed that several activities take place on the alpha and theta bands during the REM sleep phase [35]. The sleep behavior disorder treated in this paper takes place during the REM sleep. Therefore, the results of this study indicate that the affected bands of alpha and theta play an important role in distinguishing between healthy and RBD-sick patients. In a study carried out by Fantini et al. [36] 15 RBD patients and 15 healthy people are examined in a waking state and REM sleep state using EEG recordings to determine whether brain stem structures are responsible for cortical activation in RBD patients. The frequency bands have been divided into theta, delta, alpha, beta 1 and beta 2 (up to $30 \mathrm{~Hz}$ ). The RBD patients showed conspicuous features in the theta and beta 2 bands both in waking and in sleeping states.

Based on the current state of knowledge, no studies have been conducted to determine which frequency bands are necessary to identify the REM sleep 
behavior disorder. Although the sources mentioned above take analysis of RBD patients in relation to EEG data into account, various other aspects such as the risk for RBD patients of contracting further cognitive disorders, or the REM sleep phase of sick and healthy people are investigated here. A consequence is that a determination of which frequency bands could be relevant for the disorder can be made, but there are no exact investigations in this research field. In addition, it should be noted that in previous studies only frequency bands as whole bands are considered, but the method used in this paper divides the bands into $0.5 \mathrm{~Hz}$ frequency ranges. This allows a much deeper look into the individual ranges. The hypothesis of this paper was to increase the information density and quality with the help of more detailed frequency bands and thus to achieve a high accuracy for the detection of the RBD disorder. Furthermore, a fast method to detect Rapid Eye Movement sleep behavior disorder should result. By dividing the standard frequency bands into smaller ranges, this approach has been used to show that there are highly informative frequency ranges in the frequency bands of delta, theta and alpha. The achieved accuracy of 90.12 percent confirms the assumption that the subdivision into smaller frequency ranges and the consideration of 10 minutes EEGs are sufficient to achieve very good results in the identification of RBD patients. Considering the 10 most important classifiers in terms of the variable importance, four variables in the delta, three variables in the theta and three variables in the alpha range are shown. Previous studies considering the frequency ranges associated with RBD and further cognitive disorders showed conspicuous features in the theta and delta bands. With the analysis of the REM sleep phase, relevant features of alpha and theta bands could be identified. The sleep behavior disorder occurs in the REM phase, so we assumed that these two frequency bands are important for the identification of RBD. This assumption could be proven in this paper. In addition, it could be determined that the delta band plays an important role for the identification of the sleep behavior disorder. By considering the three predictive subbands, a pattern in the spectral power could be detected. Compared to the non-RBD patients, the spectral power of the RBD-patients is always stronger. Only the spectral power of the last frequency range is lower. As a result, there is a strong spectral power in the lower regions of the subbands, which decreases in higher frequency ranges. Considering all predictive frequency bands, Cohens d shows a medium/strong effect power. The results of this paper prove that it is reasonable to use this new and innovative approach to analyze the frequency bands instead of the standard division, and thus new findings from the EEG records can be obtained.

This machine learning method based on the analysis of fine-graded spectra can be used as a technical assistance for physicians. Due to this, it allows a faster evaluation with its classification and snippets of only 10 minutes length instead of eight hour recordings, but also demonstrates which $\mathrm{Hz}$ frequencies physicians should pay attention to for detecting the disorder. The shorter time span is a facilitation for the patient as well as for the attending physician. In the context of this research about the RBD disorder, early detection could have an impact on avoiding secondary diseases like Parkinson or Dementia, which strongly affect the lives of the patients [13].

\section{Conclusion}

As demonstrated in this paper, an accurate and reliable automatic classification method was implemented, as well as showing which $\mathrm{Hz}$ frequencies are particularly relevant for the classification. By analyzing fine-graded spectra instead of standard frequency bands (delta, theta, alpha, etc.) a more detailed result is possible. In this way, important frequencies become apparent which were not considered before, and open up a new perspective for the analysis. Furthermore, patterns in the spectral power of RBD patients could be determined. This work confirms previous results that information with respect to $\mathrm{RBD}$ disorder are hidden in theta and alpha bands in which REM sleep can be detected [35]. Additionally, there is a new finding. Beside theta and alpha bands, frequencies in the delta band are in particular highly relevant for classifying RBD and non-RBD data. The delta frequency band has a range of $0.5-3.5 \mathrm{~Hz}$ and is characterized by deep sleep.

\subsection{Limitations}

While the internal validity of our method was high, since we used a reliable dataset from the PhysioBank database, the method should be introduced as a medical application in a clinical environment to assess external validity. By doing this, more data of patients could be analyzed to validate the results of this research. A further limitation could be the absence of acceptance by physicians to apply the method in their daily routines.

Despite we intensively evaluated other traditional machine learning approaches such as clustering [37] and also most modern convolutional neural networks, which are outstanding in other domains such as image 
recognition [38-40], we achieved the best results here with our novel method, originally proposed in [41, 42]. However, future work should extend the application of further novel machine learning approaches.

In addition, medication and personality [43-45] influence EEG data, and as a result, our classifier. While the internal validity of our model is very high due to the rigorous $\mathrm{k}$-fold-cross-validation, improving external validity by training with additional datasets is also an important step to improve the model. Also, the influence of individual differences in brain activity on EEG e.g. other mental disorders need to be analyzed and considered in future studies.

\subsection{Future work}

In future work we will report common method bias evaluations [46, 47]. In addition, we will triangulate EEG data with other physiological sensors (i.e. electrocardiogram $[48,49]$, electrodermal activity [50, 51], eye fixation [52-54], eye pupil diameter [55-58]). Furthermore, we will experimentally evaluate whether our novel approach is also robust under various conditions of a user's cognitive workload [59-61] and related concepts [62-64], concentration [65], and mindfulness $[66,67]$. In addition, we will report results on successfully applying our novel procedure to other sleep data [68] and other diseases such as epilepsy [69] and schizophrenia [70, 71].

To assess preconditions to implement the approach in real clinical environments we will conduct an implementation study to evaluate acceptance [72-74] and trust $[75,76]$ by physicians and patients and determine if the automated approach improves the coordination [77-79] between doctors more efficiently.

In addition, future work on a sensitivity analysis of other timespans than 10 minute recordings is also indicated.

\section{Acknowledgements}

We would like to thank the anonymous reviewers and the editors, who provided very helpful comments on the refinement of the HICSS paper.

This research is partly funded by the German Federal Ministry of Education and Research (no. 13FH4E03IA, no. 13FH4E07IA, no. 13FH176PX8) and by the German Federal Ministry of Economics and Technology (EXIST program, no. 03EGSBW477).

\section{References}

[1] K. Tsoi, S. Poon, and P. Hung, Patrick, "Introduction to the Minitrack on Big Data on Healthcare Application", In
Proceedings of the 51st Hawaii International Conference on System Sciences (HICSS-51), January 3-6, Big Island, Hawaii, p. 2777, 2018.

[2] K. Tsoi, S. Poon, and P. Hung, Patrick, "Introduction to the Minitrack on Big Data on Healthcare Application", In Proceedings of the 52nd Hawaii International Conference on System Sciences (HICSS-52), January 8-11, Maui, Hawaii, p. 3720, 2019.

[3] A. Esteva, A. Robicquet, B. Ramsundar, V. Kuleshov, M. DePristo, K. Chou, C. Cui, G. Corrado, S. Thrun, and J. Dean, "A guide to deep learning in healthcare", Nature Medicine, vol. 25, pp. 24-29, 2019.

[4] C. Poon, N. Gorji, M. Latt, K. Tsoi, B. Choi, C. Loy, S. Poon, "Derivation and Analysis of Dynamic Handwriting Features as Clinical Markers of Parkinson's Disease", In Proceedings of the 52nd Hawaii International Conference on System Sciences (HICSS-52), January 8-11, Maui, Hawaii, pp. 3721-3730, 2019.

[5] S. K. Poon, J. Poon, M. McGrane, X. Zhou, P. W. H. Kwan, R. Zhang, B. Liu, J. Gao, C. Loy, K. Chan, D. Manyuen Sze, "A novel approach in discovering significant interactions from TCM patient prescription data", International Journal of Data Mining and Bioinformatics, vol. 5, no. 4, pp. 353-368, 2011.

[6] M. J. Sateia, "International Classification of Sleep Disorders-Third Edition: Highlights and Modifications", CHEST, vol. 146, no. 5, pp. 1387-1394, 2014.

[7] V. Latreille, J. Carrier, J. Montplaisir, M. Lafortune and J.-F. Gagnon, "Non-rapid eye movement sleep characteristics in idiopathic REM sleep behavior disorder", Journal of the Neurological Sciences, vol. 310, no. 1-2, pp. 159-162, 2011.

[8] B. F. Boeve, M. H. Silber, C. B. Saper, T. J. Ferman, D. W. Dickson, J. E. Parisi, E. E. Benarroch, J. E. Ahlskog, G. E. Smith, R. C. Caselli, M. Tippman-Peikert, E. J. Olson, S.-C. Lin, T. Young, Z. Wszolek, C. H. Schenck, M. W. Mahowald, P. R. Castillo, K. Del Tredici and H. Braak, "Pathophysiology of REM sleep behaviour disorder and relevance to neurodegenerative disease", Brain, vol. 130, no. 11, pp. 2770-2788, 2007.

[9] R. N. Aurora, R. S. Zak, R. K. Maganti, S. H. Auerbach, K. R. Casey, S. Chowdhuri, A. Karippot, K. Ramar, D. A. Kristo and T. I. Morgenthaler, "Best Practice Guide for the Treatment of REM Sleep Behavior Disorder (RBD)", Journal of Clinical Sleep Medicine, vol. 6, no. 1, pp. 85-95, 2010.

[10] F. Andreotti, H. Phan, N. Cooray, C. Lo, M. T. M. Hu and M. De Vos, "Multichannel Sleep Stage Classification and Transfer Learning using Convolutional Neural Networks", 40th Annual International Conference of the IEEE Engineering in Medicine and Biology Society (EMBC), Honolulu, HI, USA, pp. 171-174, 2018. 
[11] M. J. Howell and C. H. Schenck, „Rapid Eye Movement Sleep Behavior Disorder and Neurodegenerative Disease", JAMA Neurology, vol.72, no. 6, 2015, pp. 707-712.

[12] D. A. Barone and C. Henchcliffe, "Rapid eye movement sleep behavior disorder and the link to alphasynucleinopathies", Clinical Neurophysiology, vol. 129, no. 8, pp. 1551-1564, 2018.

[13] A. B. Klok, J. Edin, M. Cesari, A. N. Olesen, P. Jennum and H. B.D. Sorensen, "A new fully automated randomforest algorithm for sleep staging", 40th Annual International Conference of the IEEE Engineering in Medicine and Biology Society (EMBC), Honolulu, HI, USA, pp. 49204923, 2018.

[14] S. Siuly and Y. Li, "Designing a robust feature extraction method based on optimum allocation and principal component analysis for epileptic EEG signal classification", Computer Methods and Programs in Biomedicine, vol. 119, no. 1, pp. 29-42, 2015.

[15] R. Islam, A. Rahim, H. Akter, R. Kabir and J. Shin, "Optimal IMF Selection of EMD for Sleep Disorder Diagnosis using EEG Signals", 3rd International Conference on Applications in Information Technology, AizuWakamatsu, Japan, pp. 96-101, 2018.

[16] M. Teplan, "Fundamentals of EEG Measurement", Measurement Science Review, vol. 2, no. 2, 2002.

[17] G. R. Müller-Putz, R. Riedl and S. C. Wriessnegger, "Electroencephalography (EEG) as a Research Tool in the Information Systems Discipline: Foundations, Measurement, and Applications", Communications of the Association for Information Systems, vol. 37, no. 46, pp. 911-948, 2015.

[18] A. R. Hevner, S. T. March, J. Park and S. Ram, "Design Science in Information Systems Research", MIS Quarterly, vol. 28, no. 1, pp. 75-105, 2004.

[19] G. H. Klem, H. O. Lüders, H. H. Jasper and C. Elger, "The ten-twenty electrode system of the International Federation", Electroencephalography and Clinical Neurophysiology, vol. 52, no. 3, pp 3-6, 1999.

[20] S. P. Fitzgibbon, D. M. W. Powers, K. J. Pope and C. R. Clark, "Removal of EEG Noise and Artifact Using Blind Source Separation", Journal of Clinical Neurophysiology, vol. 24, no. 3, pp. 232-243, 2007

[21] K. Islam, A. Rastegarnia and Z. Yang, "Methods for artifact detection and removal from scalp EEG: A review", Neurophysiologie Clinique/Clinical Neurophysiology, vol. 46, no. 4-5, pp. 287-305, 2016.

[22] T.-P. Jung, C. Humphries, T.-W. Lee, S. Makeig, M. J. McKeown, V. Iragui and T. J. Sejnowski, "Extended ICA Removes Artifacts from Electroencephalographic Recordings", Advances in Neural Information Processing Systems, vol. 10, pp. 894-900, 1998.
[23] J. Muthuswamy and N. V. Thakor, "Spectral analysis methods for neurological signals", Journal of Neuroscience Methods, vol. 83, no. 1, pp. 1-14, 1998.

[24] M. Akin, "Comparison of Wavelet Transform and FFT Methods in the Analysis of EEG Signals", Journal of Medical Systems, vol. 26, no. 3, pp. 241-247, 2002.

[25] M. K. van Vugt, P. B. Sederberg, M. J. Kahana, "Comparison of spectral analysis methods for characterizing brain oscillations", Journal of Neuroscience Methods, vol. 162, no. 1-2, pp. 49-63, 2007.

[26] M. K. Ahirwal and N. D Iondhe, "Power Spectrum Analysis of EEG Signals for Estimating Visual Attention", International Journal of Computer Applications, vol. 42, no. 15, pp. 22-25, 2012.

[27] K. J. Archer and R. V. Kimes, "Empirical characterization of random forest variable importance measures", Computational Statistics \& Data Analysis, vol. 52, no. 4, pp. 2249-2260, 2008.

[28] A. Liaw and M. Wiener, "Classification and Regression by randomForest", $R$ News, vol. 2, no. 3, pp. 18-22, 2002.

[29] L. Breiman, "Random Forests" Machine Learning, vol. 45 , no. 1 , pp. 5-32, 2001.

[30] T. Fushiki, "Estimation of prediction error by using Kfold cross-validation", Statistics and Computing, vol. 21, no. 2, pp. 137-146, 2011.

[31] Y. Bengi and, Y. Grandvalet, "No Unbiased Estimator of the Variance of K-Fold Cross-Validation", Journal of Machine Learning Research, vol. 5, pp. 1089-1105, 2004.

[32] J. D. Rodriguez, A. Perez and J. A. Lozano, "Sensitivity Analysis of k-Fold Cross Validation in Prediction Error Estimation", IEEE Transactions on Pattern Analysis and Machine Intelligence, vol. 32, no. 3, pp. 569 - 575, 2010.

[33] J. Peever, P. H. Luppi and J. Montplaisir, "Breakdown in REM sleep circuitry underlies REM sleep behavior disorder", Trends in Neurosciences, vol. 37, no. 5, pp. 279288, 2014.

[34] J. F. Gagnon, R. B. Postuma, S. Mazza, J. Doyon and J. Montplaisir, "Rapid-eye-movement sleep behaviour disorder and neurodegenerative diseases", The Lancet Neurology, vol.5, no. 5, pp. 424-432, 2006.

[35] B. F. Boeve, M. H. Silber and T. J. Ferman, "REM Sleep Behavior Disorder in Parkinson's Disease and Dementia With Lewy Bodies“, Journal of Geriatric Psychiatry and Neurology, vol. 17, no. 3, pp. 146-157, 2004.

[36] M. L. Fantini, J. F. Gagnon, D. Petit, S. Rompré, A. Décary, J. Carrier and J. Montplaisir, "Slowing of electroencephalogram in rapid eye movement sleep behavior disorder", Annals of Neurology, vol. 53, no. 6, pp. 774-780, 2003. 
[37] F. Baumann, D. Emmert, H. Baumgartl, and R. Buettner, "Hardcore Gamer Profiling: Results from an unsupervised learning approach to playing behavior on the Steam platform", Procedia Computer Science, vol. 126, pp. 1289-1297, 2018.

[38] R. Buettner, and H. Baumgartl "A highly effective deep learning based escape route recognition module for autonomous robots in crisis and emergency situations", HICSS-52 Proceedings, pp. 659-666, 2019.

[39] H. Baumgartl, and R. Buettner, "Development of a highly precise place recognition module for effective humanrobot interactions in changing lighting and viewpoint conditions", HICSS-53 Proceedings, 2020, in press.

[40] H. Baumgartl, J. Tomas, R. Buettner, and M. Merkel, “A novel Deep-Learning Approach for Automated NonDestructive Testing in Quality Assurance based on Convolutional Neural Networks", ACEX-2019 Proceedings, 2019 , in press

[41] R. Buettner, T. Rieg, and J. Frick, "Machine Learning based Diagnosis of Diseases Using the Unfolded EEG Spectra: Towards an Intelligent Software Sensor", in Information Systems and Neuroscience, vol. 32 of LNISO, Springer, 2019, in press.

[42] T. Rieg, J. Frick, M. Hitzler, and R. Buettner, "Highperformance detection of alcoholism by unfolding the amalgamated EEG spectra using the Random Forests method", In HICSS-52 Proceedings, pp. 3769-3777.

[43] R. Buettner, "Predicting user behavior in electronic markets based on personality-mining in large online social networks: A personality-based product recommender framework," Electronic Markets, vol. 27, no. 3, pp. 247-265, 2017.

[44] R. Buettner, "Innovative Personality-based Digital Services”, PACIS 2016 Proceedings, 2016.

[45] R. Buettner, "Personality as a predictor of business social media usage: An empirical investigation of XING usage patterns", PACIS 2016 Proceedings, 2016.

[46] R. Buettner, "Getting a job via career-oriented social networking markets: The weakness of too many ties", Electronic Markets, vol. 27, no. 4, pp. 371-385, 2017.

[47] R. Buettner, "Analyzing the Problem of Employee Internal Social Network Site Avoidance: Are Users Resistant due to their Privacy Concerns?", HICSS-48 Proceedings, pp. 1819-1828, 2015.

[48] R. Buettner, L. Bachus, L. Konzmann, and S. Prohaska, "Asking Both the User's Heart and Its Owner: Empirical Evidence for Substance Dualism", Information Systems and Neuroscience, vol. 29, pp. 251-257, 2018.
[49] R. Buettner, and M. Schunter, "Efficient machine learning based detection of heart disease", Proc. IEEE Healthcom 2019, in press.

[50] R. Buettner, “Asking both the User's Brain and its Owner using Subjective and Objective Psychophysiological NeuroIS Instruments", ICIS 2017 Proceedings, 2017.

[51] A. Eckhardt, C. Maier, and R. Buettner, "The Influence of Pressure to Perform and Experience on Changing Perceptions and User Performance: A Multi-Method Experimental Analysis”, ICIS 2012 Proceedings, 2012.

[52] A. Eckhardt et al., "Objective measures of IS usage behavior under conditions of experience and pressure using eye fixation data", ICIS 2013 Proceedings, 2013.

[53] R. Buettner, "A user's cognitive workload perspective in negotiation support systems: An eye-tracking experiment", PACIS 2016 Proceedings, 2016.

[54] R. Buettner, "Cognitive Workload of Humans Using Artificial Intelligence Systems: Towards Objective Measurement Applying Eye-Tracking Technology”, LNAI, vol. 8077, pp. 37-48, 2013.

[55] R. Buettner, "Social inclusion in eParticipation and eGovernment solutions: A systematic laboratoryexperimental approach using objective psychophysiological measures", LNI Proc., vol. P-221, pp. 260-261, 2013.

[56] R. Buettner, "Investigation of the Relationship Between Visual Website Complexity and Users' Mental Workload: A NeuroIS Perspective", Information Systems and Neuroscience, vol. 10, pp. 123-128, 2015.

[57] R. Buettner, B. Daxenberger, A. Eckhardt, and C. Maier, "Cognitive Workload Induced by Information Systems: Introducing an Objective Way of Measuring based on Pupillary Diameter Responses", SIGHCI 2013 Proc., 2013.

[58] R. Buettner, S. Sauer, C. Maier, and A. Eckhardt, "Towards ex ante Prediction of User Performance: A novel NeuroIS Methodology based on Real-Time Measurement of Mental Effort", HICSS-48 Proc., pp. 533-542, 2015.

[59] R. Buettner, "Analyzing Mental Workload States on the Basis of the Pupillary Hippus", in NeuroIS '14 Proc., p. 52, 2014.

[60] R. Buettner, "Investigation of the Relationship Between Visual Website Complexity and Users' Mental Workload: A NeuroIS Perspective", in Information Systems and Neuro Science, vol. 10 of LNISO, pp. 123-128, 2015.

[61] R. Buettner, S. Sauer, C. Maier, and A. Eckhardt, "Realtime Prediction of User Performance based on Pupillary Assessment via Eye Tracking", AIS Trans Hum.-Comput Interact, vol. 10, no. 1, pp. 26-56, 2018. 
[62] R. Buettner, "The relationship between visual website complexity and a user's mental workload: A NeuroIS perspective", in Information Systems and Neuro Science, vol. 16 of LNISO, pp. 107-113, Springer, 2016.

[63] R. Buettner, "A user's cognitive workload perspective in negotiation support systems: An eye-tracking experiment", in PACIS 2016 Proc.

[64] R. Buettner, I. J. Timm, I. F. Scheuermann, C. Koot, and M. Roessle, "Stationarity of a user's pupil size signal as a precondition of pupillary-based mental workload evaluation", in Information Systems and Neuro Science, vol. 25 of LNISO, pp. 195-200, Springer, 2017.

[65] R. Buettner, H. Baumgartl, and D. Sauter, "Microsaccades as a predictor of a user's level of concentration", in Information Systems and Neuroscience, vol. 29 of LNISO, pp. 173-177, Springer, 2018.

[66] S. Sauer, J. Lemke, W. Zinn, R. Buettner, and N. Kohls, "Mindful in a random forest: Assessing the validity of mindfulness items using random forests methods", Pers Individ Differ, vol. 81, pp. 117-123, 2015.

[67] S. Sauer, R. Buettner, T. Heidenreich, J. Lemke, C. Berg, and C. Kurz, "Mindful Machine Learning: Using Machine Learning Algorithms to Predict the Practice of Mindfulness", Eur J Psychol Assess, vol. 34, no. 1, pp. 6-13, 2018.

[68] R. Buettner, J. Fuhrmann, and L. Kolb, "Towards highperformance differentiation between Narcolepsy and Idiopathic Hypersomnia in 10 minute EEG recordings using a Novel Machine Learning Approach," in IEEE Healthcom 2019 Proc., in press.

[69] R. Buettner, T. Rieg, and J. Frick, "High-performance detection of epilepsy in seizure-free EEG recordings: A novel machine learning approach using very specific epileptic EEG sub-bands", in ICIS 2019 Proc., in press.
[70] R. Buettner, M. Hirschmiller, K. Schlosser, M. Roessle, M. Fernandes, and I. J. Timm, "High-performance exclusion of schizophrenia using a novel machine learning method on EEG data”, in IEEE Healthcom 2019 Proc., in press.

[71] R. Buettner, D. Beil, S. Scholz, and A. Djemai, "Development of a machine learning based algorithm to accurately detect schizophrenia based on one-minute EEG recordings", HICSS-53 Proceedings, in press.

[72] R. Buettner, B. Daxenberger, and C. Woesle, "User acceptance in different electronic negotiation systems - a comparative approach", in ICEBE 2013 Proc., pp. 1-8, 2013.

[73] R. Buettner, "Towards a New Personal Information Technology Acceptance Model: Conceptualization and Empirical Evidence from a Bring Your Own Device Dataset", in AMCIS '15 Proc., 2015.

[74] R. Buettner, "Getting a Job via Career-oriented Social Networking Sites: The Weakness of Ties", in HICSS-49 Proc., pp. 2156-2165, 2016.

[75] F. Meixner and R. Buettner, "Trust as an Integral Part for Success of Cloud Computing", in ICIW 2012 Proc., pp. 207-214, 2012

[76] R. Buettner, "Cooperation in Hunting and Food-sharing: A Two-Player Bio-inspired Trust Model", in BIONETICS '09 Proc., pp. 1-10, 2009.

[77] R. Buettner, "A Classification Structure for Automated Negotiations," in WI-IAT 2006 Proc., pp. 523-530, 2006.

[78] R. Buettner, "The State of the Art in Automated Negotiation Models of the Behavior and Information Perspective,” ITSSA, vol. 1, no. 4, pp. 351-356, 2006.

[79] I. Timm, L. Reuter, J. O. Berndt, A.-S. Ulfert, T. Ellwart, and C. Antoni, "Analyzing the Effects of Role Configuration in Logistics Processes using Multiagent-Based Simulation: An Interdisciplinary Approach," in HICSS-52 Proc., pp. 5476-5485, 2019. 